\title{
BESCHREIBUNG UND ZEICHNERISCHE AUFNAHME DES MINARETTS VON ÉRD AUS DEM JAHRE 1857
}

\author{
PÉTER PROHÁSZKA
}

H-2500 Esztergom, József Attila tér 2.

E-mail:prohaszkapeter@freemail.hu

\begin{abstract}
This paper deals with the description and drawing prepared in 1857 on the minaret of Érd. The k. k. CentralCommission zur Erforschung und Erhaltung der Baudenkmale was established on the territory of the Habsburg Monarchy in 1850. The conservers, the correspondents and the engineers of the k. k. Baubehörde surveyed and pictured several architectural and archaeological monuments and reported on them to Vienna. In this study a drawing and descriptions of the minaret standing at the border of Érd are published. The survey was made for the order of Florian Menapace, Landesbaudirektor, who sent the drawing and the attached remarks to Vienna. Only the drawing can be found nowadays in the Archiv of the Central-Commission, today the Planarchiv of the Bundesdenkmalamt. The drawing was made by Wenzl Kansky. Fortunately, the description prepared by the engineer Alajos Zalay and the Menapace report based on it remained in Hungarian State Archiv in Budapest. They are important sources on the minaret built in the $17^{\text {th }}$ century and the autor gives an evaluation on it in the study.
\end{abstract}

Keywords: Minaret of Érd, archivs, Ottoman Period, architectural and archaeological monuments

\section{EINFÜHRUNG}

Die in Ungarn nach dem Niederschlag des ungarischen Freiheitskampfes von 1848/1849 eingeführte absolutistische Politik wirkte auch auf die Bodendenkmalpflege, und zwar positiv aus, als die 1850 in Wien gegründete und mit amtlichen Befugnissen ausgestatte k. k. Central-Commission zur Erforschung und Erhaltung der Baudenkmale ihre Tätigkeit in Ungarn aufnahm. Obwohl Kaiser Franz Joseph die Vorlage über ihre Gründung bzw. ihre Statuten schon 1850 ratifizierte, setzte ihre Tätigkeit erst 1853 ein. ${ }^{1}$ Die Kommission wirkte bis zum Ausgleich neben dem Ungarischen Königreich auch in Siebenbürgen und auf dem Gebiet der sog. Militärgrenze. ${ }^{2}$ Eine unverzichtbare Voraussetzung ihrer Tätigkeit war der Ausbau eines Netzwerks von politisch zuverlässigen und für die Baudenkmäler bzw. archäologischen Funde aufgeschlossenen Personen, die als Konservatoren und Korrespondenten ehrenamtlich die Baudenkmäler und die zum Vorschein gekommenen Funde verzeichneten, aufnahmen und beschrieben. ${ }^{3}$ Diese Personen sandten ihre Berichte oft mit Zeichnungen und Vermessungen illustriert nach Wien, welche aber nur zu einem kleinen Teil in den Jahrbüchern der Central-Commission sowie in der Fundchronik von

${ }^{1}$ Für die sprachliche Korrektur des vorliegenden Textes bedanke ich mich bei Prof. Dr. Hubert Emmerig. Über die Umstände der Errichtung der Central-Commission und ihre frühe Tätigkeit siehe: FRODEL 1988, 60-76.
${ }^{2} \mathrm{Zu}$ ihrer Tätigkeit in Ungarn: MARosI 1983, 13-18; MEZEY-SZENTESI 1996, 47-67.

${ }^{3}$ MeZEy-SzEnTEsi 1996, 51-54. 


\section{Hisureset \\ ter Lirt ( itterrsoseteg)}

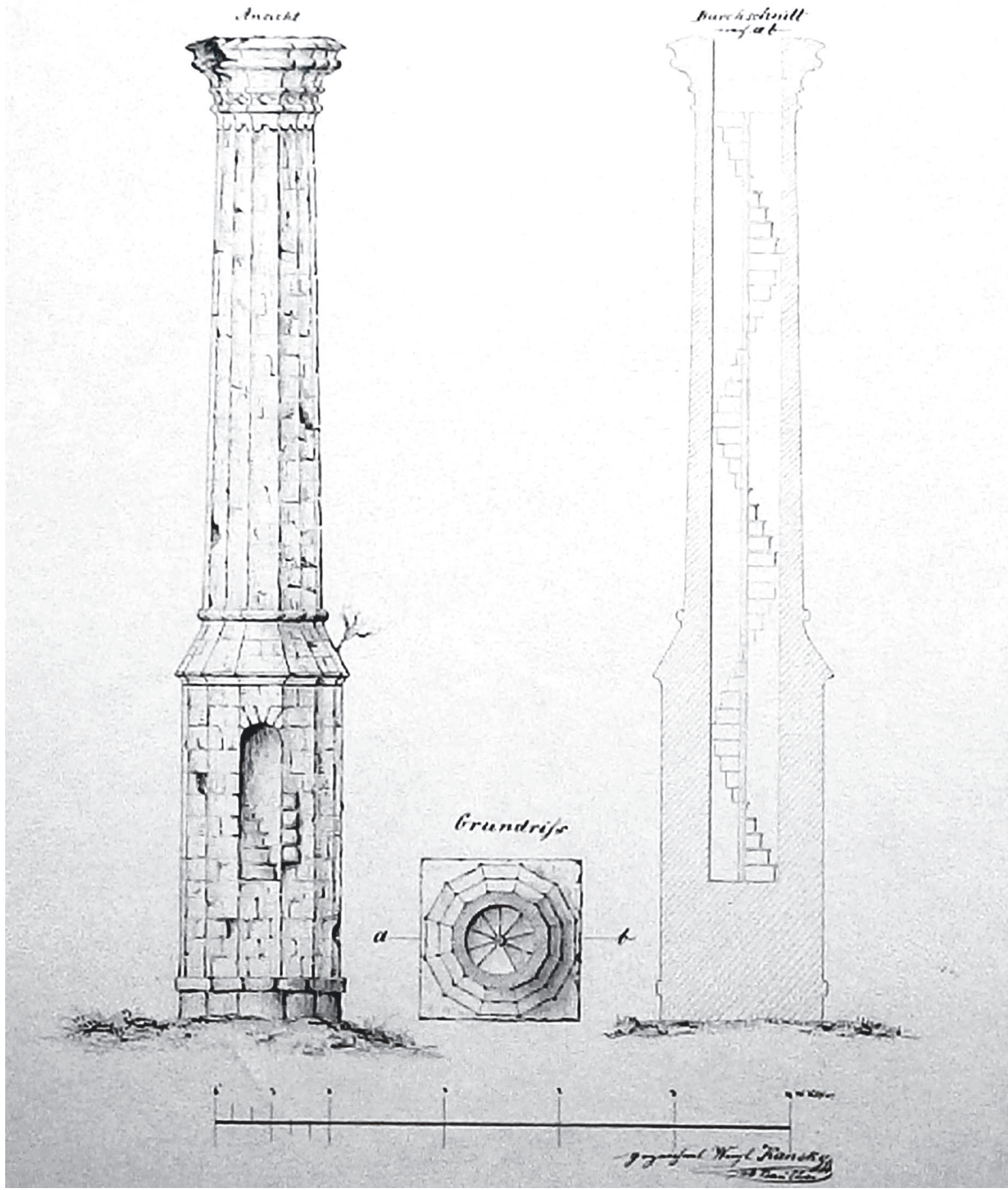

Abb. 1. Die Vermessungszeichnung von Wenzl Kansky (Bundesdenkmalamt, Planarchiv, Wien) Acta Archaeologica Academiae Scientiarum Hungaricae 65, 2014 
Seidl und Kenner veröffentlicht wurden. Der größte Teil des Akten- und Zeichnungsmaterials ging leider im Laufe des 20. Jahrhunderts zugrunde, was ein schwerer Verlust für die archäologische und Denkmalforschung war. ${ }^{4} \mathrm{Im}$ Planarchiv des Wiener Bundesdenkmalamtes befinden sich noch solche Zeichnungen von Baudenkmälern und Gebäuden, die der Central-Commission noch vor dem Ausgleich geschickt wurden. ${ }^{5}$ Unter diesen befindet sich eine gefärbte Vermessungszeichnung über das Minarett in der Gemarkung des Dorfes Érd (Komitat Pest) (Abb. 1). ${ }^{6}$ Diese Zeichnung wurde von dem Bauingenieurassistenten Wenzl Kansky 1857 angefertigt. ${ }^{7}$ Über das Bauwerk befindet sich bloß eine kurze Nachricht im Jahresbericht der Central-Commission: „Auf Ersuchen der Central-Commission erklärte sich S. E. der Herr Baron Sina bereit, die Baudenkmale zu Érd und Trentsin nach den gegebenen Andeutungen erhellen zu wollen. " ${ }^{8}$ Diese Zeichnung stammte aber gewiss nicht vom Baron Sina, worauf der in der linken Ecke befindliche Stempel mit Inventarnummer hinweist. Nach dem Stempel K. K. BAU DIRECTOR FÜR UNGARN wurde sie als Beilage zur Akte 655/1857 vom k. k. Baudirektor für Ungarn nach Wien geschickt. Die Aktenstücke kamen aber bei den Forschungen in Wien nicht zum Vorschein. Eine Lösung brachte die Durchsicht des Aktenmaterials der k. k. Landesbaubehörde für Ungarn. ${ }^{9}$

Die Führung der Central-Commission musste schnell einsehen, dass die Anwerbung von interessierten, hoch angesehenen, gebildeten und auch politisch zuverlässigen Personen nur sehr langsam und umständlich voranging. Daher versuchte das Handelsministerium, die schon verfügbaren Personen und Behörden für die Erhaltung und Aufnahme der Baudenkmale heranzuziehen. So wurden am 24. Juni 1853 von allerhöchster Stelle die „Instruktionen für die k. k. Baubeamten bezüglich der Erhaltung der Baudenkmale“ ausgegeben, ${ }^{10}$ damit die schon bestehenden Strukturen und die damit verbundenen qualifizierten Personen genutzt werden konnten. ${ }^{11}$ Die 1850 organiserten k. k. Baubehörden sorgten mit ihren Beamten für die Planung, Errichtung und Instandsetzung von Straßen und öffentlichen Gebäuden. Nach den Paragraphen 5 und 6 der Instruktionen gehörte zu ihren Aufgaben nunmehr auch die Aufnahme und Anfertigung von Plänen oder Zeichnungen derjenigen Baudenkmale, die ihnen entweder bezeichnet wurden oder nach ihrem eigenen Ermessen als sehr beachtenswert erschienen. ${ }^{12}$ In dieser Hinsicht ist es nicht überraschend, dass den Jahresberichten und Sitzungsprotokollen nach anfänglich die meisten Zeichnungen und Berichte von ihnen eingegangen sind. ${ }^{13}$ Florian Menapace, Direktor der Landesbaubehörde, war Leiter und Antreiber dieser Forschungen in Ungarn, der aus dem italienischen Trient wegen seiner kaisertreuen Haltung zuerst nach Siebenbürgen, dann nach Ungarn versetzt wurde. Er sandte der Central-Commission zahlreiche Zeichnungen und Berichte, die von ihm kompiliert bzw. verfasst waren. ${ }^{14}$ Dank seiner Position und Verfügungen haben die Baubeamten und -ingenieure zahlreiche Baudenkmäler und archäologische Fundorte aufgenommen und gezeichnet. Seine Wirksamkeit wurde von der Central-Commission schnell mit der Ernennung zum Korrespondenten anerkannt. ${ }^{15}$

Bei Durchsicht des Aktenmaterials der Landesbaubehörde im Ungarischen Staatsarchiv kamen solche Akten zum Vorschein, in denen sich Verfügungen über das Minarett zu Érd befinden. In einer Akte liegen eine Beschreibung des Minaretts vom Ingenieur Alois Zalay und ein Konzept des Berichts des Landesbaudirektors. Beide Aktenstücke zusammen mit der Zeichnung sind wichtige Quellen zur Forschungsgeschichte des Minaretts von Érd, die hier erstmals veröffentlicht werden.

\footnotetext{
${ }^{4}$ SZENTESI 1991, 48-49.

${ }^{5}$ Zahlreiche Zeichnungen wurden von E. Szentesi veröffentlicht: SZENTESI 2012, 2-29.

${ }^{6}$ An dieser Stelle möchte ich mich bei Herrn Hans Scheifele (BDA Planarchiv Wien) bedanken, der meine Forschungen im Planarchiv unterstützte und die Veröffentlichung der Zeichnung bewilligte. Die Recherchen in den Wiener Archiven konnte ich mit der Unterstützung des Collegium Hungaricum Stipendiums durchführen.

${ }^{7}$ Zum Verfertiger der Zeichnung: MEZEY-SZENTESI 1996, 51

${ }^{8}$ Bericht über die Tätigkeit der k. k. Central-Commission in der Periode vom 1. Juli 1857 bis Ende September 1858. Jahrbuch der kaiserl. königl. Central-Commission zur Erforschung und Erhaltung der Baudenkmale 3 (1859) XII.

${ }^{9}$ Über die Tätigkeit der Baudirektion: EMBER 1942-1945, $360-373$.
}

${ }^{10}$ Instructionen für die K. K. Baubeamten bezüglich der Erhaltung der Baudenkmale. Jahrbuch der kaiserl. königl. CentralCommission zur Erforschung und Erhaltung der Baudenkmale 1 (1856) 28-32.

${ }^{11}$ SZENTESI 2012, 7-8.

${ }^{12}$ Instructionen für die K. K. Baubeamten bezüglich der Erhaltung der Baudenkmale. Jahrbuch der kaiserl. königl. CentralCommission zur Erforschung und Erhaltung der Baudenkmale 1 (1856) 29-30.

${ }^{13}$ Sitzungs-Protokolle der k. k. Central-Commission zur Erforschung und Erhaltung der Baudenkmale (im amtlichen Auszuge) (Jahre 1853-1857). Wien 1858.

${ }^{14}$ MEZEY-SZENTESI 1996, 53.

${ }^{15}$ SZENTESI 2012, 11. 
Florian Menapace bat in seinem Brief vom 3. Juli 1857 den Ingenieur Alois Zalay, den Leiter des Komitatsbauamtes zu Székesfehérvár (Stuhlweißenburg), um die Beauftragung des Ingenieurs Scheutzer, dass er Zeichnungen des Minaretts machen solle. ${ }^{16}$ Wie Menapace erwähnte, habe schon Herr Pöschel im Laufe des vergangenen Jahres die Aufnahme des Gebäudes versprochen, was aber nicht geschehen sei. Menapace gab auch die Ansichten der Zeichnung vor bzw. bat um eine schnelle Erledigung, weil er sowohl die Zeichnung als auch die Beschreibung mit dem Jahresbericht zusammen der Central-Commission senden wolle. ${ }^{17}$ Zalay war jene Person, die Menapace drei Jahre vorher - in seinem Bericht vom 26. Dezember 1854 - auf das Minarett aufmerksam gemacht und zugleich um Instruktionen gebeten hatte. ${ }^{18}$ So ist es nicht überraschend, dass Zalay nicht nur die Zeichnung machen ließ, sondern dem Landesbaudirektor damit auch eine kurze Beschreibung des Minaretts sandte. ${ }^{19}$

\section{Kurze Beschreibung \\ des Minarets außerhalb Érd im Weißenburger Comitate}

Von den wenigen Denkmaler, welche man der einstigen Größe der ottomanischen Macht in Ungarn eine geringe Spur liefern, dürfte das zu Érd bestehende bescheidene Minaret eines der besterhaltenen sein.

Da die Türken im Jahre 1526 am 31. August bis Stuhlweissenburg vorgedrungen und dieser befestigten Stadt eben so wie am $11^{\mathrm{t}}$ September desselben Jahres sich auch der Stadt u. Festung Ofen bemächtigt haben, so dürfte die Erbauung des besagten Leutt-oder Wachthurmes in die nächst darauffolgenden Jahren erfolgt sein.

Da der Ort Érd auch Hamsabeg genannt wird, so dürfte es keinem Zweifel obliegen, daß der frägliche Thurm auf Anordnung des ansehnlichen türkischen Heerführers Hamsabeg erfolgt sei.

Dieser Thurm ist aus Werkstücken des nahe liegenden Sóskuter Steinbruches rein und nutt erbauet, einst seine Höhe von $812^{\circ}$, der Würfel des Postaments in ' $1^{\circ}-3^{\prime}-0$ ”, hat 49 Stück Stufen, welche eine massive Treppe bilden, und die Werkstücke aus hartem canchylicum Kalk sind mit Eisen Klammern sorgfältigst verbauet, so zwar daß dieses Thürmchen bei einigen Ausbesserung noch eine weitere Dauer von 327 Jahren verspricht, zumal die auf den Thurme ersichtlichen Schaden mehr dem Muthwillen als der zerstörend einwirkenden Zeit zugeschrieben werden müssen.

Gegen Osten schien eine kleine Kammer angebracht gewesen zu sein, wie die hervorstehenden Verbindungsquadern dieß bezeugen. Am Gipfel des Thurmes ist eine Brust-Mäuerchen von circa 2', und eine breite Stufe, welche zugleich als Ruhe Platz gedient hätte.

Dieses zwar höchst einfache, jedoch nicht ganz geschmacktes erbaute Thürmchen durfte bei einer Reparatur noch für sehr lange Zeit als ein bleibendes Denkmal der einstigen Größe der ottomanischen Macht Ausbreitung, den Hamsabeger Hügel zieren.

Stuhlweißenburg am 7 July/857

Alois Zalay

Weil Menapace sowohl die Zeichnung als auch die Beschreibung zur Verfügung hatte, fasste er ihren Inhalt in seinem Bericht von 18. Juli 1857 zusammen, in dem er der Central-Commission seine Vorschläge über die Bewahrung und Instandsetzung des Bauwerks mitteilte. ${ }^{20}$

Obgleich die Errichtung des in der Zeichnung F aufgenommenen Minaret's im Dorfe Érd /Hamsabeg/auf der Ofener-Eszekier Staatsstraße bei der Verzweig der Route Stuhlweissenburg-Graz erst in den Jahren 1526 - bis 30 fallen durfte, so glaube ich von diesem türkischen Denkmal um so mehr eine Zeichnung machen zu laßen, als

\footnotetext{
${ }^{16}$ Ein Konzept seines Briefes befindet sich im Aktenmaterial: Ungarisches Staatsarchiv (Budapest) D 238 ANr. 592/1857.

${ }^{17}$ Ungarisches Staatsarchiv (Budapest) D 238 ANr. 592/1857: „... die Zeichnung in Ansicht - Durchschnitte und Grundriß - mit thunlichster Beschleunigung anher zu senden, da der Gefertigte dem Jahresbericht, wo die Zeichnung und Beschreibung des besprochenen Minaretts auch vorkommt, längstens bis 9. dieses absenden muß.“
}

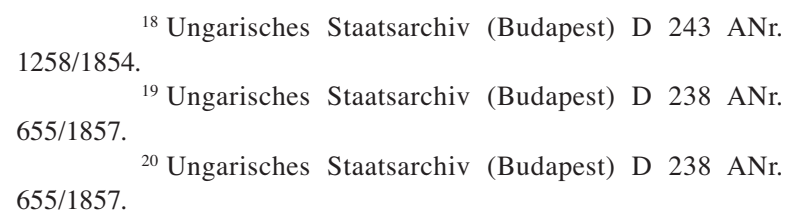
$1258 / 1854$.

${ }^{19}$ Ungarisches Staatsarchiv (Budapest) D 238 ANr. $655 / 1857$. $655 / 1857$. 
meinerseits die Benennung des Hügels, worauf dieses Thürmchen stehet - der Hamsabeger Hügel - von dem so bekannten türkischen Heerführer Hamsabeg', welcher nach der Sage, bei der Vertreibung der Türken zurückgeblieben, zu Érd gewohnt und daselbst begraben seyn soll, herrühret, andererseits weil dieses türkische Monument durchgängig aus Werkstücken des naheliegenden Sóskut'er Steinbruches rein und mit besonderer Genauigkeit erbaut ist, und dessen innere maßive, mit 49 Stufen versehene Wendeltreppe mit den behauenen Werkstücken ein regelmäßiges, mit kaum sichtbaren Mörtelverband gefugtes Ganzes bildet.

Dieses aus sehr dauerhaftem Gestein erbauete und vermög seiner festen und kunstgerechten Ausfiihrung noch dermalen, als von dem Zahn der Zeit nicht viel angegriffene Thürmchen ragt über 50 Schuh aus dem Erde hervor.

Gegen Osten scheint eine kleine Kammer angebracht gewesen zu sein, wie das die hervorstehenden Verbindungsquader bezeugen. Am Gipfel bestehet ein Brustwehr-Mäuerchen von circa 3'Schuh hohe.

Das zwar höchst einfach, jedoch nicht ganz geschmacktes erbauete Minaret verrath, bei der Sorgfalt womit es erbaut wurde, und bei dem zur genauen Verbindung der Werkstücke verwendeten Fließ, daß es wirklich nicht zu den gewöhnlichen Zwecken errichtet war, gleichzeitig deutet aus dem erhoheten Terrain um demselben hervorstehenden Zokel, daß die ursprüngliche Höhe nur größere war.

Es wäre wunschenswerth, das Erdreich an dieser Stelle zu besichtigen und das Thürmchen in dessen früheren Gestalt zu erhalten, der Baron - Sina'sche - Gödöllö- und Ercsier Herrschaft als Eigenthümer dürfte unschwer werden, die Freistellung durch Terrainabgrabung so wie die sei, und würde nöthige Restaurationen des Hamsabeg'er Minarets bewirken zu lassen.

\section{DAS MINARETT VON ÉRD IM SPIEGEL DER BESCHREIBUNG VON ALOIS ZALAY UND DER ZEICHNUNG VON WENZL KANSKY}

Nur wenige Baudenkmäler der fast 150-jährigen Türkenherrschaft haben sich in Ungarn erhalten, und diese sind meistens nicht mehr intakt bzw. zerstört. ${ }^{21}$ Ein Teil davon sind sakrale Bauten, die Minarette und Moscheen. Die Anmerkung von Alois Zalay ist zutreffend: Das in der Gemarkung von Érd stehende Minarett gehöre wirklich zu den ,besterhaltenen“, obwohl die zu ihm gehörende Moschee nach der Vertreibung der Türken vollständig abgerissen wurde. Der Turm könnte seine Erhaltung dem Umstand verdanken, dass er mit seinem 15 m Höhe als Wachturm diente. Außer Érd stehen noch Minarette in Fünfkirchen, Szigetvár und Eger, bei denen der spitzige Bedeckung ebenfalls zugrunde ging. ${ }^{22}$ Eine Zeichnung des Ingenieurs János Varsányi vom Minarett zu Érd erschien in Magyar Akadémiai Értesítő schon 1847 (Abb. 2), aber János Érdy beschäftigte sich in seinem Beitrag nur mit den urzeitlichen Hügeln von Százhalombatta, nicht mit dem Bauwerk. ${ }^{23}$ Obwohl in manchen Arbeiten darüber berichtet wurde, ${ }^{24}$ geschah seine archäologische und architektonische Erforschung erst in der ersten Hälfte der 1960er Jahre unter der Leitung von Géza Fehér, woraufhin auch eine denkmalpflegerische Instandsetzung erfolgte $(A b b .3) .{ }^{25}$

Nach dem Feldzug im Jahre 1541 geriet die Siedlung Érd unter türkische Herrschaft und wurde wegen ihrer strategischen Lage Teil der türkischen Aufmarsch- und Nachschubroute. Der zweite, bis heute genutzte Name des Ortes Hamzsabég stammt ebenfalls aus diesen Zeiten. Er kommt von dem türkischen Feldherrn des 16. Jahrhunderts mit dem Namen Hamza, Beg von Székesfehérvár, zu dessen Besitz der Ort gehörte. Er ließ hier auf Befehl Sulejmans I. eine Plankenburg errichten. ${ }^{26}$ Diese Plankenburg hatte anfänglich eine kleine Besatzung, erst wegen der Feldzüge im 15-jährigen Krieg wurde die Zahl der Soldaten erhöht. Deswegen wurde es nötig, eine Moschee und das dazugehörige Minarett zu errichten, damit die Soldaten ihre Religion hier ausüben konnten. ${ }^{27}$ An den Beg als Bauherrn des Minaretts dachten sowohl Zalay als auch Menapace - sie irrten sich jedoch, da sein Aufbau erst im 17. Jahrhundert erfolgte. ${ }^{28}$ Am nördlichen Rand des Siedlungsteils Ófalu von Érd steht auch heute noch das ungefähr 15 m hohe Minarett, das samt seiner Umgebung zwischen 1962 und 1965 archäologisch erforscht wurde.

${ }^{21}$ Siehe die Zusammenfassungen: GERŐ 1976; GERŐ 1980.

${ }^{22}$ MOLNÁR 1961, 13.

${ }^{23}$ LUTZENBACHER 1847, 286-287. Im Text befand sich folgende Anmerkung (285): ,... faragott kövekből épített török torony” [aus behauenen Steinen gebauter türkischer Turm].

${ }^{24}$ VoIT 1958, 349-350; MolnÁR 1965, 142-147.

${ }^{25}$ FEHÉR 1963, 66-67; FEHÉr 1964, 74.
${ }^{26}$ MOLNÁR 1965, 142-144.

${ }^{27}$ MolNÁR 1965, 142-143.

${ }^{28}$ Über die unterschiedlichen Meinungen siehe: MoLNÁR 1965, 142-147. Die archäologischen Beobachtungen belegen seine Errichtung im 17. Jahrhundert. Die Dokumentation der Ausgrabung von G. Fehér befindet sich im Archiv des Ungarischen Nationalmuseums III.111/1963, X.250/1963, IX.102/1964. 


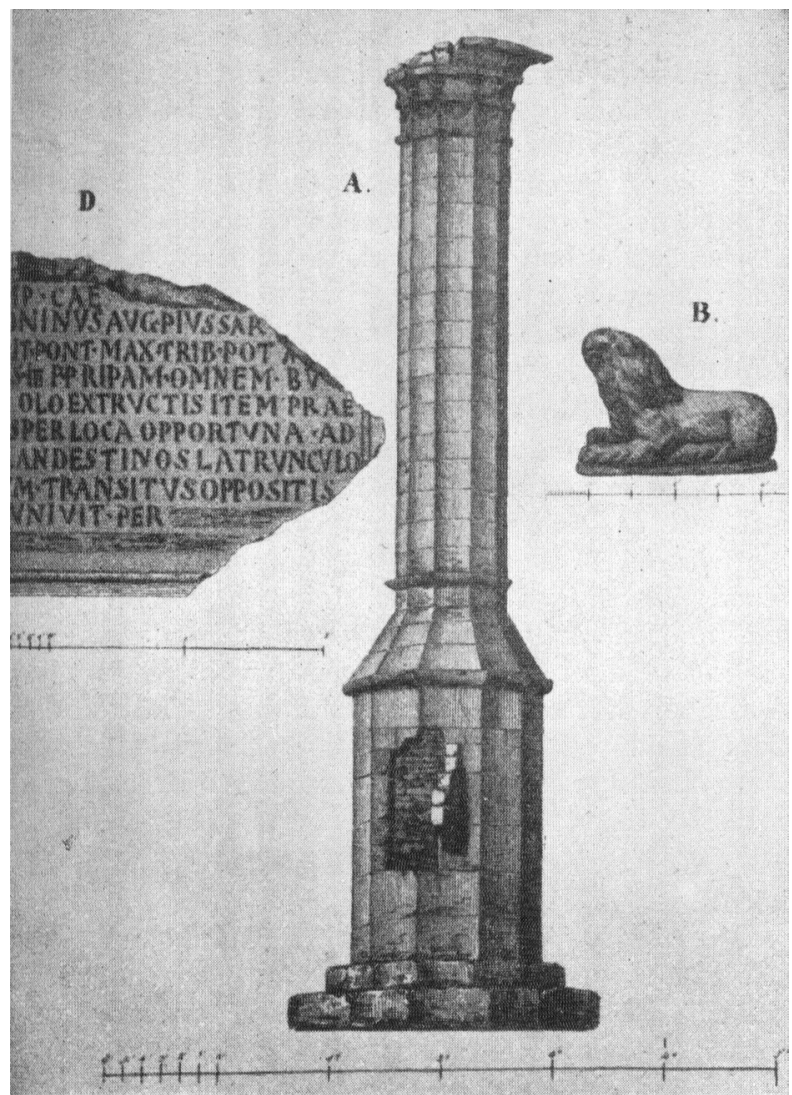

Abb. 2. Die Zeichnung von János Varsányi über das Minarett von Érd (LuTZENBACHER 1847, 286)

Ziel dieser Untersuchung war die Klärung des Moscheeumrisses und die Gewinnung von baugeschichtlichen Angaben. Das Minarett wurde - ähnlich wie die anderen in Ungarn - aus hiesigem Steinmaterial errichtet; ${ }^{29}$ wie bereits Zalay erwähnte, war die Quelle wirklich der Steinbruch von Sóskút. Das Minarett steht auf einem würfelförmigen Steinhocker, dessen Größe auch in den Berichten angegeben wurde. Das wird auch durch die Zeichnung von Kansky bestätigt. ${ }^{30}$ Das auffallend niedrige und stämmige Bauwerk unterscheidet sich in seiner strukturellen und formalen Ausbildung kaum von den anderen Minaretten. Seine auf der Zeichnung gut sichtbare, stämmige Sockelwand hat aber gegenüber den anderen einen zwölfeckigen Grundriss. An seiner östlichen Seite befindet sich eine Rundbogentüröffnung in einer Höhe von ungefähr $2 \mathrm{~m}$. Von hier läuft eine Wendeltreppe mit 50 (nicht 49) Stufen zum Rundbalkon. ${ }^{31}$ Der zwölfseitige Turmkörper ist $8 \mathrm{~m}$ hoch und steht auf einem trapezförmigen Sockel. ${ }^{32}$ Der Rundbalkon hat ebenfalls einen zwölfeckigen Grundriss; der darüber befindliche schlanke Korb sowie der mit einem Halbmond verzierte Hut gingen zugrunde. ${ }^{33}$ Ihre Überreste wurden bei der archäologischen Ausgrabung nicht gefunden. Zalay erwähnt, dass nach Osten eine kleine Kammer angebaut gewesen sei, was hervorstehende Verbindungsquader belegten. Diese Beobachtung ist insofern stichhaltig, als bei der Ausgrabung die Spuren eines $9,8 \times$ 9,8 m großen, zur östlichen Seite an das Minarett anschließenden Bauwerks beobachtet wurden, das aber keine Kammer, sondern die Moschee war. ${ }^{34}$ Wahrscheinlich konnte man das Minarett vom Balkon der Moschee aus betreten. ${ }^{35}$ Die Wand ist unter dem Vorsatz des Balkons mit eselrücken- bzw. herzförmigen Verzierungen geschmückt. ${ }^{36}$

\footnotetext{
${ }^{29}$ MolNÁr 1965, 13.

${ }^{30}$ Dies wurde durch die Ausgrabung von G. Fehér bestätigt und war vorher unbekannt: VoIGT 1958, 356 und 357, Abb. 293. J. Molnár vermutete das nur: MOLNÁR 1965, 144.

${ }^{31}$ Molnár 1965, 145.

${ }^{32}$ MolNÁr 1961, 145.
}

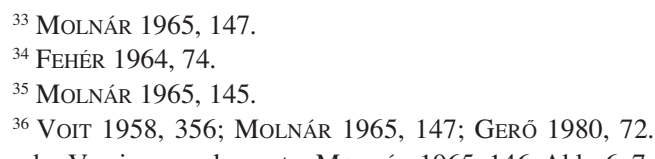
Eine Skizze der Verzierungselemente: MolnÁR 1965, 146, Abb. 6-7. 


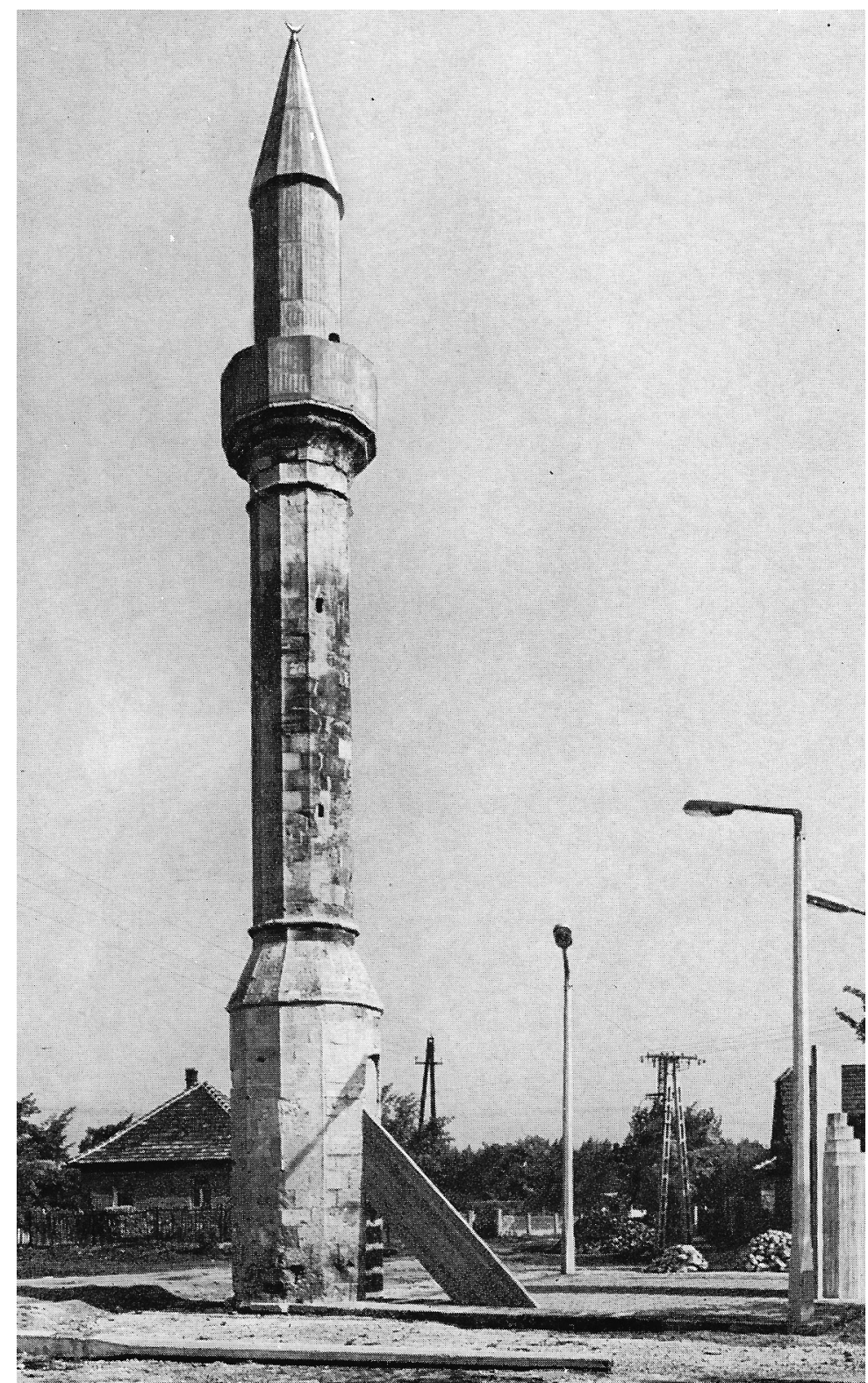

Abb. 3. Das Minarett von Érd nach der denkmalpflegerischen Instandsetzung

Auf die Bedeutung der Verzierungen machte József Molnár aufmerksam, der auch ihre Motive rekonstruierte. ${ }^{37}$ Diese Verzierungen sind, wenn auch stilisiert, auf der Zeichnung von Kansky dargestellt. Im Hinblick auf die gründliche Beschreibung und die Zeichnung können wir nur bedauern, dass diese weder in den Mitteilungen noch im Jahrbuch der Central-Commission veröffentlicht wurden.

Obwohl der Vorschlag von Florian Menapace bezüglich der Geländeplanierung und Sanierung des Minaretts nicht verwirklicht wurde, zeugen die im Planarchiv des Wiener Bundesdenkmalamtes befindliche Zeichnung und die im Nationalarchiv verwahrten Berichte davon, dass der Landesbaudirektor sich gemäß den Instruktionen um Aufnahme und Erhaltung der Baudenkmäler bemühte.

${ }^{37}$ MolnÁR 1961, 18. 


\section{LITERATUR}

EMBER 1942-1945

FEHÉR 1963

FEHÉR 1964

FRODEL 1988

\section{GERŐ 1976}

GERŌ 1980

LUTZENBACHER 1847

MAROSI 1983

MeZEy-SZEnTESI 1996

MoLNÁR 1961

MoLNÁR 1965

SZENTESI 1991

SZENTESI 2012

VoIT 1958
= Gy. EMBER: A magyarországi építészeti igazgatóság történetének vázlata [Abriss der Geschichte der ungarischen Landesbaubehörde]. LK 20-23 (1942-1945) 360-373.

= G. FeHÉR: Érd-Ófalu. RégFüz I/16. Budapest 1963, 66-67.

= G. FeHÉR: Érd-Ófalu. RégFüz I/17. Budapest 1964, 74.

= W. FRODEL: Idee und Verwirklichung. Das Werden der staatlichen Denkmalpflege in Österreich. Köln-Graz 1988

= GY. GERŐ: Turkish Monuments in Hungary. Budapest 1976

= Gy. GERō: Az oszmán-török építészet Magyarországon (Dzsámik, türbék, fürdők) [Die osmanischtürkische Architektur in Ungarn. Moscheen, Türben, Bäder]. MüF 12. Budapest 1980.

= J. LutZenBAChER: Pogány magyar sírok [Heidnische ungarische Gräber]. Magyar Akadémiai Értesítő 7 (1847) 282-289.

= E. MARosI: Die Anfänge der Denkmalpflege und die Tätigkeit der k.u.k. Zentralkommission in Ungarn. In: E. Marosi (Hrsg.): Die ungarische Kunstgeschichte und die Wiener Schule 1846-1930. Ausstellung, Collegium Hungaricum, Wien, September 1983. Budapest 1983, 13-18.

= A. D. MezeY-E. SzENTEsi: Az állami múemlékvédelem kezdetei Magyarországon. A Central-Commission zur Erforschung und Erhaltung der Baudenkmale magyarországi működése [Die Anfänge der staatlichen Denkmalpflege in Ungarn. Die Tätigkeit der Central-Commission zur Erforschung und Erhaltung der Baudenkmale in Ungarn]. In: I. Bardoly-A. Haris (Hrsg.): A magyar müemlékvédelem korszakai. Tanulmányok [Die Epochen der ungarischen Denkmalpflege. Beiträge]. Budapest 1996, 47-67.

= J. MolNÁR: A hazai minarék esztétikai vizsgálatai [Die ästhetische Untersuchung der heimischen Minaretten]. MüÉ 10 (1961) 11-15.

= J. MolnÁR: Hamza bég dzsámija Érden [Die Moschee des Begs Hamza in Érd]. Müemlékvédelem 8/3 (1965) 142-147.

= E. SzENTESI: Bekezdések a magyarországi műemlékvédelem előtörténetéből. I: Egy kérés a CentralCommission magyarországi vonatkozású anyagi ügyeiben [Absätze aus der Vorgeschichte der ungarischen Denkmalpflege. I: Ein Gesuch in Sachen von ungarischen Materialien der CentralCommission]. Múemlékvédelmi Szemle 1991/2, 48-49.

= E. SzENTESI: Az intézményes műemlékvédelem kezdetei Magyarországon [Die Anfänge der institutionellen Denkmalpflege in Ungarn]. Mủemlékvédelem 56/1-2 (2012) 2-29.

= P. VorT: Érd. Mecset út. Minaret. In: D. Dercsényi (Hrsg.): Magyarország mủemléki topográfiája. Pest megye múemlékei I. [Die Kunstdenkmäler des Komitats Pest I. Die Kunsttopographie Ungarns] Budapest 1958, 349-350. 